Research Paper

\title{
The PLCE1 rs2274223 variant is associated with the risk of laryngeal squamous cell carcinoma
}

\author{
Yi Zhang ${ }^{\circledR}$, Wei Li$^{2}$, Ying Wang 3 , Ningyu Wang ${ }^{1^{凶}}$ \\ 1. Department of Otolaryngology-Head and Neck Surgery, Beijing Chaoyang Hospital, Capital Medical University, Beijing, China. \\ 2. The Department of Head and Neck surgery, Hubei Cancer Hospital, Wuhan, Hubei, China. \\ 3. Department of Pathology, Beijing Chaoyang Hospital, Capital Medical University, Beijing, China. \\ $\triangle$ Corresponding authors: Dr. Yi Zhang, ORCID ID: 0000-0002-6126-8014, Department of Otolaryngology-Head and Neck Surgery, Beijing Chaoyang Hospital, \\ Capital Medical University, No.8 Gongtinanlu, 100020, Beijing, China. E-mail: dr.yizhang@ccmu.edu.cn; Dr. Ningyu Wang, ORCID ID: 0000-0001-8260-3582, \\ Department of Otolaryngology-Head and Neck Surgery, Beijing Chaoyang Hospital, Capital Medical University, No.8 Gongtinanlu, 100020, Beijing, China. \\ E-mail: wny85231645@163.com.
}

(c) The author(s). This is an open access article distributed under the terms of the Creative Commons Attribution License (https://creativecommons.org/licenses/by/4.0/). See http://ivyspring.com/terms for full terms and conditions.

Received: 2020.06.02; Accepted: 2020.09.16; Published: 2020.10.08

\begin{abstract}
Background: Laryngeal squamous cell carcinoma (LSCC) ranks second in the mortality rate in respiratory malignant tumors and has potential similarity in genomic alterations with the esophageal squamous cell carcinoma (ESCC). The PLCE1 rs2274223 variant is the most significant susceptibility loci identified in ESCC. Whether it is also associated with LSCC susceptibility is still unclear.

Materials and Methods: A total of 331 LSCC patients and 349 healthy controls were recruited in this study. The PLCEl rs2274223 variant was genotyped by using the Taqman SNP Genotyping Assay. Association between PLCEl rs2274223 variant and LSCC risk was estimated by logistic regression analysis, which was performed using SAS software.

Results: The PLCE1 rs2274223 variant was identified to be significantly associated with the susceptibility of LSCC in the additive model $(\mathrm{OR}=1.40,95 \% \mathrm{Cl}: 1.06-1.86, P=0.019)$. Compared with the wild-type (AA) carriers, the risk genotype (GG) carriers had a 2.8 -fold risk of LSCC ( $95 \% \mathrm{Cl}$ : 1.13-7.06, $P=0.026)$. Stratified analysis showed that the association between rs2274223 and LSCC risk was with higher significance in individuals above $60(P=0.027)$ males $(P=0.030)$ or non-smokers $(P=0.026)$.

Conclusion: The PLCE1 rs2274223 variant was significantly associated with risk of LSCC, which may be a potential biomarker and therapeutic target for the LSCC.
\end{abstract}

Key words: Laryngeal squamous cell carcinoma (LSCC), single nucleotide polymorphism (SNP), PLCE1, rs2274223

\section{Introduction}

Laryngeal cancer is one of most common head and neck cancers, the majority of cases are laryngeal squamous cell carcinoma (LSCC) [1]. This tumor type ranks second in the mortality rate in respiratory malignant tumors $[2,3]$. Genetic and environmental factors contribute to the disease, and genetic variation is one of the most promoting factors that accounting for its pathogenesis [4, 5]. Another squamous cell carcinoma (SCC) in the upper aerodigestive tract, the esophageal squamous cell carcinoma (ESCC), has many similarities in the genomic alteration and susceptibility genes with LSCC.

Phospholipase C epsilon 1 gene (PLCE1) is a C isoenzyme of phosphorus esterase, which was first identified in C. elegant by Shibatohge $\mathrm{M}$ and colleges [6], and its gene is located in chromosome 10q23 in human [7]. It plays an important role in cell growth, differentiation and oncogenesis [8]. Different expression profiles of PLCE1, like overexpression or down-regulation, could be found in different types of cancer, and functioned in diverse manners [9-12]. Large scale genome-wide association (GWAS) studies from different groups had demonstrated that the rs2274223, located in exon 26 of PLCE1, was strongly associated with the risk of ESCC [13-17]. The PLCE1 polymorphisms and its distribution have been 
reported to be involved in its gene expression and tumor development [18]. The PLCE1 rs2274223 is of particular interest and attention for its association with susceptibility of many types of cancer, like colorectal cancer [19], gastric cancer [18, 19], esophageal cancer, and several other digestive tract cancers [8, 20-24]. Although the PLCE1 rs2274223 has been reported to affect many types of digestive tract cancers, whether the PLCE1 rs2274223 A/G variant is associated with LSCC susceptibility is still unclear.

The above summary suggested that role of PLCE1 and rs2274223 had been extensively studied in diverse cancer types, especially in ESCC. In the present study, we genotyped the PLCE1 rs2274223 A/G polymorphism with 331 LSCC patients and 349 healthy controls and analyzed the association between the PLCE1 rs2274223 and LSCC susceptibility. Our work will give novel insights into the PLCE1 rs2274223 variant as a biomarker for the early detection of LSCC.

\section{Materials and Methods}

\section{Study subjects}

In this study, we enrolled 331 LSCC patients and 349 healthy controls from Hubei Cancer Hospital and Beijing Chaoyang Hospital between March 2013 to December 2018. The LSCC patients were diagnosed histopathologically by at least two pathologists according to the World Health Organization classification. The controls were cancer-free individuals who seeking routine physical examination in the same hospital as patients were recruited. All participants had written informed consent at recruitment. There is about $45 \%$ power to identify significant results with estimated odds ratio being 1.40 and the minor allele frequency of variant in controls being 0.20 . The demographic characteristics like sex, age, smoking and drinking status were obtained from the medical records. The study was approved by Hubei Cancer Hospital Ethics Committee with KYLLB2017002.

\section{SNP genotyping}

Blood samples from LSCC patients and healthy controls were collected and stored at -80 Celsius degree, and the whole genome DNA were extracted for genotyping analysis by using TIANamp Genomic DNA Kit (Tiangen Biotech, China). The PLCE1 rs2274223 picked up from ESCC GWAS analysis was selected for genotyping, and the variant was genotyped by using the Taqman SNP Genotyping Assay (Applied Biosystems, USA).

\section{Statistical analysis}

Logistic regression analysis adjusted for sex, age, smoking and drinking status were used to assess the strength of association between the PLCE1 rs2274223 and LSCC risk. All statistical analyses were performed using SAS software (version 9.1; SAS Institute, Cary, NC) and SNPStats app [25]. A $P$ value $<0.05$ was considered as statistically significant and all tests were two-sided.

\section{Results}

\section{Study sample processing and the characteristics of subjects}

The basic demographic, smoking and drinking status of LSCC patients and healthy controls are listed in the Table 1. The current study recruited 331 LSCC patients and 349 healthy controls, with mean age of 60.42 and 62.02, respectively. There are 308 males and 23 females in LSCC group, 295 males and 54 females in healthy control group. Smoking and drinking factors are thought to be involved in gene mutation and cancer development. Therefore, we also collected and listed the related information of subjects in our study (Table 1).

\section{The PLCE1 rs2274223 genotypes associates with the susceptibility of LSCC}

In our study, the PLCE1 rs2274223 was selected for genotyping. The number and the percentage of LSCC patients with AA, AG and GG genotypes were $178(53.8 \%), 135(40.8 \%)$ and $18(5.4 \%)$, respectively (Table 2). In healthy control group, these data were AA, 216 (61.9\%), AG 125 (35.8\%), and GG, 8 (2.3\%), respectively (Table 2). The Hardy-Weinberg balance analysis showed that the allelic distributions in cases and controls were in equilibrium $(P>0.01)$. The odds ratio (OR) and corresponding 95\% confidence intervals (CIs) adjusted for sex, age, smoking and drinking status for each genotype was used to assess the associations between PLCE1 rs2274223 and LSCC risk by using unconditional multivariate logistic regression analysis. The OR, 95\% CIs, and the corresponding $p$ value to each genotype were listed in Table 3. The results showed that when comparing to AA genotype carriers, the GG genotype carriers were significantly associated with higher LSCC risk with OR being 2.82 (95\% $\mathrm{CI}=1.13-7.06, P=0.026)$. Besides, by using the additive model, the results demonstrated that the rs2274223 G allele carriers was significantly associated with higher LSCC risk comparing to the rs2274223 A allele carriers with OR being 1.40 (95\% CI $=1.06-1.86, P=0.019)$ (Table 3 ). 


\section{Stratified analysis showed the association between PLCE1 rs2274223 and LSCC risk was with higher significance in individuals elder 60 , males or non-smokers}

The above data indicated that the PLCE1 rs2274223 variant was significantly associated with LSCC risk. As subject characteristics like age, sex, smoking and drinking status are all cancer-related factors [26-28], we next tested the associations by stratified each of the above factors (Table 3). The results suggested that the association between the PLCE1 rs2274223 and LSCC risk was with higher significance in individuals elder than $60(\mathrm{OR}=1.50$, $95 \% \mathrm{CI}=1.05-2.15, P=0.027)$, males $(\mathrm{OR}=1.39,95 \% \mathrm{CI}$ $=1.03-1.86, P=0.030)$ or nonsmokers $(\mathrm{OR}=2.00,95 \%$ $\mathrm{CI}=1.09-3.69, P=0.026)$. The association between the PLCE1 rs2274223 and LSCC risk were not significance in individuals less than 60 years old, females or smokers. The association between the PLCE1 rs2274223 and LSCC risk were not significance in both drinkers and nondrinkers, which may due to relatively small sample size in this study. No significant interaction was observed for rs 2274223 and sex, age, smoking status or drinking status (Table 4).

Table 1. Characteristics summary of study subjects

\begin{tabular}{llll}
\hline & LSCC $(\mathrm{n}=331)$ & Controls $(\mathrm{n}=349)$ & $P$ values* \\
\hline $\begin{array}{l}\text { Age (years), Mean } \pm \text { SD } \\
\text { Gender, } \mathrm{n}(\%)\end{array}$ & $60.42 \pm 10.00$ & $62.02 \pm 8.61$ & \\
$\begin{array}{l}\text { Male } \\
\text { Female }\end{array}$ & $308(93.1)$ & $295(84.5)$ & 0.0004 \\
$\begin{array}{l}\text { Smoking status } \\
\text { Nonsmoker, } \mathrm{n}(\%)\end{array}$ & $23(6.9)$ & $54(15.5)$ & \\
$\begin{array}{l}\text { Smoker, } \mathrm{n}(\%) \\
\text { Drinking status }\end{array}$ & $26(13.9)$ & $145(41.5)$ & $<0.0001$ \\
$\begin{array}{l}\text { Nondrinker, } \mathrm{n}(\%) \\
\text { Drinker, } \mathrm{n}(\%)\end{array}$ & $111(33.5)$ & $204(58.5)$ & \\
\hline
\end{tabular}

$P$ values are calculated by using chi-squared test.

\section{Discussion}

Investigation of tumor susceptibility is of particular significance for cancer evaluation and therapy [29], and many SNPs had been extensively confirmed to be associated with tumor susceptibility [30-34]. Our current study focused on the association between LSCC risk and PLCE1 rs2274223, a very hot and promising biomarker for upper digestive cancers, especially ESCC.

After collecting 331 LSCC patients and 349 healthy controls samples and genotyped with PLCE1 rs2274223, we found that the PLCE1 rs2274223-G was significantly associated with a higher LSCC risk, which is similar with its association in ESCC [13-17]. Further stratified analysis demonstrated that age, sex, and smoking status may affect the association. However, the PLCE rs2274223variant exhibited different behaviors in the susceptibility of different cancer types. For example, the PLCE1 rs2274223 A/G might reduce gene expression and the $\mathrm{G}$ allele might contribute to a higher risk of colorectal cancer [19]. And the bladder cancer development was suggested to be related to increased PLCE1 expression [9], and overexpression of PLCE1 also positively affected on transfer of the head and neck squamous cell carcinoma [10]. Therefore, whether and how the PLCE1 rs2274223 altered the PLCE1 expression paten in LSCC patients and the LSCC development need to be investigated in the future.

There were still several limitations in this study at current stage. Firstly, the sample size of this study was relatively small with limited power. More subjects should be recruited in the future study to replicate the association. Besides, subjects from different region may exhibit different sensitivity, so large multicenter study should be performed, and more comprehensive analysis should be carried out. Finally, cancer development was always driven by multiple genes mutations, so analysis of PLCE1 rs2274223 combining with other SNP on affecting LSCC susceptibility should be performed in following study.

In summary, this study identified that the rs2274223 variant in PLCE1 was significantly associated with the susceptibility of LSCC. This variant may serve as a potential biomarker for the early detection of LSCC.

\section{Abbreviations}

PLCE1: phospholipase C epsilon 1; LSCC: laryngeal squamous cell carcinoma; OR: odds ratio; 95\% CI: 95\% confidence interval; GWAS: genomewide association study; SNP: single nucleotide polymorphism.

Table 2. Allele frequencies and exact test for Hardy-Weinberg equilibrium of rs 2274223

\begin{tabular}{|c|c|c|c|c|c|c|}
\hline & A/A Count (Proportion) & A/G Count (Proportion) & G/G Count (Proportion) & A Count (Proportion) & G Count (Proportion) & $P$ value \\
\hline All subjects & $394(0.58)$ & $260(0.38)$ & $26(0.04)$ & $1048(0.77)$ & $312(0.23)$ & 0.039 \\
\hline Controls & $216(0.62)$ & $125(0.36)$ & $8(0.02)$ & $557(0.80)$ & $141(0.20)$ & 0.045 \\
\hline Cases & $178(0.54)$ & $135(0.41)$ & $18(0.05)$ & $491(0.74)$ & $171(0.26)$ & 0.310 \\
\hline
\end{tabular}

$P$ values are calculated by using exact test for Hardy-Weinberg equilibrium. 
Table 3. Summary of PLCE1 rs2274223 and its association with risk of LSCC

\begin{tabular}{|c|c|c|c|c|c|}
\hline Model & Genotype & Controls & Cases & OR $(95 \% \mathrm{CI})$ & $\begin{array}{l}P \\
\text { value }\end{array}$ \\
\hline & $\mathrm{A} / \mathrm{A}$ & 216( & $178(53.8 \%)$ & 1.00 & \\
\hline & A/G & 125 & 135 & $1.28(($ & 0.146 \\
\hline & G/G & 8 & 18( & 2.82 & 026 \\
\hline \multirow[t]{2}{*}{ Dominant } & $\mathrm{A} / \mathrm{A}$ & $216(6$ & $178(5$ & 1.00 & 0.056 \\
\hline & A/G-G/G & 133( & $153(46.2 \%)$ & $1.37(0.99-1.90)$ & \\
\hline \multirow[t]{2}{*}{ Recessive } & $\mathrm{A} / \mathrm{A}-\mathrm{A} / \mathrm{G}$ & 341 & $313(9$ & 1.00 & 0.035 \\
\hline & $\mathrm{G} / \mathrm{G}$ & $8(2.3 \%)$ & $18(5.4 \%)$ & $2.56(1.03-6.34)$ & \\
\hline \multirow[t]{2}{*}{ Overdominant } & A/A-G/G & $224(64.2 \%)$ & $196(59.2 \%)$ & 1.00 & 0.260 \\
\hline & $\mathrm{A} / \mathrm{G}$ & $125(35.8 \%)$ & $135(40.8 \%)$ & $1.21(0.87-1.68)$ & \\
\hline Log-additive & --- & --- & --- & $1.40(1.06-1.86)$ & 0.019 \\
\hline
\end{tabular}

OR, odds ratio; $\mathrm{CI}$, confidence interval. $P$ values are two sided and were calculated in logistic regression analysis adjusted for sex, age, smoking status and drinking status. Significant results were in bold.

Table 4. Summary of stratified analysis of associations between rs2274223 and LSCC risk in additive model

\begin{tabular}{lcllll}
\hline & $\begin{array}{l}\text { MAF } \\
\text { Cases }\end{array}$ & $\begin{array}{l}\text { MAF } \\
\text { Controls }\end{array}$ & OR (95\% CI) & $P$ value* & $\begin{array}{l}\text { Interaction } \\
P \text { value }\end{array}$ \\
\hline Age, years & & & & & \\
$>60$ & $\mathbf{0 . 2 7 1}$ & $\mathbf{0 . 1 9 7}$ & $\mathbf{1 . 5 0}(\mathbf{1 . 0 5 - 2 . 1 5 )}$ & $\mathbf{0 . 0 2 7}$ & 0.677 \\
$\leq 60$ & 0.245 & 0.214 & $1.23(0.75-2.00)$ & 0.408 & \\
Gender & & & & & \\
Male & $\mathbf{0 . 2 5 5}$ & $\mathbf{0 . 1 9 7}$ & $\mathbf{1 . 3 9}(\mathbf{1 . 0 3 - 1 . 8 6 )}$ & $\mathbf{0 . 0 3 0}$ & 0.798 \\
$\begin{array}{l}\text { Female } \\
\text { Smoking status }\end{array}$ & 0.304 & 0.231 & $1.80(0.56-5.80)$ & 0.323 & \\
$\begin{array}{l}\text { Smoker } \\
\text { Nonsmoker }\end{array}$ & 0.254 & 0.208 & $1.31(0.94-1.82)$ & 0.110 & 0.483 \\
$\begin{array}{l}\text { Drinking status } \\
\text { Drinker }\end{array}$ & 0.268 & 0.208 & $1.45(0.99-2.14)$ & 0.057 & 0.945 \\
Nondrinker & 0.239 & 0.197 & $1.38(0.89-2.15)$ & 0.153 &
\end{tabular}

MAF, minor allele frequency; $\mathrm{OR}$, odds ratio; $\mathrm{CI}$, confidence interval. ${ }^{*} \mathrm{P}$ values are two sided and were calculated in logistic regression analysis adjusted for sex, age, smoking status and drinking status. The interaction $P$ values were calculated by conducting a one degree-of-freedom Wald test of a single interaction parameter as implemented in an unconditional logistic regression with age, sex, smoking status or drinking status as covariates.

\section{Acknowledgments}

\section{Funding}

This study received no specific grant from any funding agency in the public, commercial or not-forprofit sectors.

\section{Authors' contributions}

Ningyu Wang conceived the idea, Yi Zhang, Wei $\mathrm{Li}$, Ying Wang enrolled the subjects, collected the sample and clinical information, Wei Li performed the DNA extraction and genotyping, Ying Wang analyzed data and prepared the tables, Yi Zhang drafted the manuscript, and all authors reviewed and final approved the manuscript.

\section{Competing Interests}

The authors have declared that no competing interest exists.

\section{References}

1. Greenlee RT, Hill-Harmon MB, Murray T, Thun M. Cancer statistics, 2001. CA Cancer J Clin. 2001; 51: 15-36.

2. Siegel RL, Miller KD, Jemal A. Cancer statistics, 2016. CA Cancer J Clin. 2016; 66: 7-30.

3. Zhang H, Zhao X, Wang M, Ji W. Key modules and hub genes identified by coexpression network analysis for revealing novel biomarkers for larynx squamous cell carcinoma. J Cell Biochem. 2019; 120: 19832-40.

4. Soskolne CL, Jhangri GS, Siemiatycki J, Lakhani R, Dewar R, Burch JD, et al. Occupational exposure to sulfuric acid in southern Ontario, Canada, in association with laryngeal cancer. Scand J Work Environ Health. 1992; 18: 225-32.

5. Muscat JE, Wynder EL. Tobacco, alcohol, asbestos, and occupational risk factors for laryngeal cancer. Cancer. 1992; 69: 2244-51.

6. Shibatohge M, Kariya K, Liao Y, Hu CD, Watari Y, Goshima M, et al. Identification of PLC210, a Caenorhabditis elegans phospholipase C, as a putative effector of Ras. J Biol Chem. 1998; 273: 6218-22.

7. Wing MR, Bourdon DM, Harden TK. PLC-epsilon: a shared effector protein in Ras-, Rho-, and G alpha beta gamma-mediated signaling. Mol Interv. 2003; 3: 273-80.

8. Xue W, Zhu M, Wang Y, He J, Zheng L. Association between PLCE1 rs2274223 A > G polymorphism and cancer risk: proof from a meta-analysis. Sci Rep. 2015; 5: 7986.

9. Ou L, Guo Y, Luo C, Wu X, Zhao Y, Cai X. RNA interference suppressing PLCE1 gene expression decreases invasive power of human bladder cancer T24 cell line. Cancer Genet Cytogenet. 2010; 200: 110-9.

10. Ma H, Wang LE, Liu Z, Sturgis EM, Wei Q. Association between novel PLCE1 variants identified in published esophageal cancer genome-wide association studies and risk of squamous cell carcinoma of the head and neck. BMC Cancer. 2011; 11: 258.

11. Wang M, Zhang R, He J, Qiu L, Li J, Wang Y, et al. Potentially functional variants of PLCE1 identified by GWASs contribute to gastric adenocarcinoma susceptibility in an eastern Chinese population. PLoS One. 2012; 7: e31932.

12. Luo XP. Phospholipase $C$ epsilon- 1 inhibits p53 expression in lung cancer. Cell Biochem Funct. 2014; 32: 294-8.

13. Wang LD, Zhou FY, Li XM, Sun LD, Song X, Jin Y, et al. Genome-wide association study of esophageal squamous cell carcinoma in Chinese subjects identifies susceptibility loci at PLCE1 and C20orf54. Nat Genet. 2010; 42: 759-63.

14. Abnet CC, Freedman ND, Hu N, Wang Z, Yu K, Shu XO, et al. A shared susceptibility locus in PLCE1 at 10q23 for gastric adenocarcinoma and esophageal squamous cell carcinoma. Nat Genet. 2010; 42: 764-7.

15. Song $\mathrm{X}, \mathrm{Li} \mathrm{WQ}, \mathrm{Hu} \mathrm{N}$, Zhao XK, Wang Z, Hyland PL, et al. GWAS follow-up study of esophageal squamous cell carcinoma identifies potential genetic loci associated with family history of upper gastrointestinal cancer. Sci Rep. 2017; 7: 4642.

16. Wei $\mathrm{Q}, \mathrm{Yu}$ D, Liu M, Wang M, Zhao M, Liu M, et al. Genome-wide association study identifies three susceptibility loci for laryngeal squamous cell carcinoma in the Chinese population. Nat Genet. 2014; 46: 1110-4.

17. Wu C, Hu Z, He Z, Jia W, Wang F, Zhou Y, et al. Genome-wide association study identifies three new susceptibility loci for esophageal squamous-cell carcinoma in Chinese populations. Nat Genet. 2011; 43: 679-84.

18. Malik MA, Srivastava P, Zargar SA, Mittal B. Phospholipase C epsilon 1 (PLCE1) haplotypes are associated with increased risk of gastric cancer in Kashmir Valley. Saudi J Gastroenterol. 2014; 20: 371-7.

19. Ezgi O, Merve A, Hakan YT, Gul O. Genetic Variations in Phospholipase C-epsilon 1 (PLCE1) and Susceptibility to Colorectal Cancer Risk. Biochem Genet. 2016; 54: 826-9.

20. Li X, Li X, Jiang M, Tian W, Zhou B. Single Nucleotide Polymorphisms in PLCE1 for Cancer Risk of Different Types: A Meta-Analysis. Front Oncol. 2018; 8: 613.

21. Guo LY, Yang N, Hu D, Zhao X, Feng B, Zhang Y, et al. PLCE1 rs2274223 polymorphism and susceptibility to esophageal cancer: a meta-analysis. Asian Pac J Cancer Prev. 2014; 15: 9107-12.

22. Duan F, Song C, Dai L, Cui S, Zhang X, Zhao X. Novel functional variants locus in PLCE1 and susceptibility to digestive tract cancer in the Chinese population: a meta-analysis. Int J Biol Markers. 2014; 29: e301-9.

23. Wang J, Lin L, Wang HQ, Chen N. PLCE1 rs2274223 polymorphism contributes to risk of esophageal cancer: evidence based on a meta-analysis. Tumour Biol. 2014; 35: 6925-31.

24. Zhang X, Zhang $Y$, Gu D, Cao C, Zhang $Q, X u$ Z, et al. Increased risk of developing digestive tract cancer in subjects carrying the PLCE1 rs2274223 A>G polymorphism: evidence from a meta-analysis. PLoS One. 2013; 8: e76425.

25. Sole X, Guino E, Valls J, Iniesta R, Moreno V. SNPStats: a web tool for the analysis of association studies. Bioinformatics. 2006; 22: 1928-9. 
26. McCormack VA, Boffetta P. Today's lifestyles, tomorrow's cancers: trends in lifestyle risk factors for cancer in low- and middle-income countries. Ann Oncol. 2011; 22: 2349-57.

27. Dossus L, Benusiglio PR. Lobular breast cancer: incidence and genetic and non-genetic risk factors. Breast Cancer Res. 2015; 17: 37.

28. Guo LY, Zhang S, Suo Z, Yang CS, Zhao X, Zhang GA, et al. PLCE1 gene in esophageal cancer and interaction with environmental factors. Asian Pac J Cancer Prev. 2015; 16: 2745-9.

29. Innocenti F, Cox NJ, Dolan ME. The use of genomic information to optimize cancer chemotherapy. Semin Oncol. 2011; 38: 186-95.

30. Imyanitov EN, Togo AV, Hanson KP. Searching for cancer-associated gene polymorphisms: promises and obstacles. Cancer Lett. 2004; 204: 3-14.

31. Shlien A, Malkin D. Copy number variations and cancer susceptibility. Curr Opin Oncol. 2010; 22: 55-63.

32. Imyanitov EN. Gene polymorphisms, apoptotic capacity and cancer risk. Hum Genet. 2009; 125: 239-46.

33. Chung CC, Chanock SJ. Current status of genome-wide association studies in cancer. Hum Genet. 2011; 130: 59-78.

34. Srinivasan S, Clements JA, Batra J. Single nucleotide polymorphisms in clinics: Fantasy or reality for cancer? Crit Rev Clin Lab Sci. 2016; 53: 29-39. 